\title{
Fabrication of Organic Thin Films by Dual Molecular Beam Deposition with Excimer Laser
}

\author{
Hiroyuki Fuchigami, Yukiyasu Nakao, Tetsuyuki Kurata, Sei Tsunoda, \\ Hiroyuki Niino* and Akira Yabe* \\ Advanced Technology R\&D Center, Mitsubishi Electric Corporation, 8-1-1 \\ Tsukaguchi Hon-machi, Amagasaki, Hyogo 661, Japan \\ *National Institute of Materials and Chemical Research, 1-1 Higashi, Tsukuba, \\ Ibaraki 305, Japan
}

Keywords: organic molecular beam deposition, intermolecular reaction, photoisomerization

\section{Introduction}

In order to utilize unique physical properties of organic materials, thin film fabrication by vapor deposition is one of the most promising techniques. However, in case of sublimation of organic materials having low vapor pressure, they are often decomposed at high temperatures. For overcoming the problem on thermal decomposition of organic materials, vapor deposition polymerization methods have been investigated to produce functional polymeric thin films. $[1,2,3]$ The polymerization resulted from the spontaneous reactions between two kinds of monomers in the ground state. We have developed a new technique of vapor deposition by combining $\mathrm{KrF}$ excimer laser-induced chemical reactions and organic molecular beam deposition (OMBD). Using this method, we obtained the films of a bis(styryl)benzene derivative, which could not be fabricated by usual vapor deposition method, through the cis-to-trans photoisomerization during the deposition.[4,5] Moreover, we performed thin film fabrication using dual molecular beams in order to induce an intermolecular reaction of hetero-molecules. In this paper, the chemical reaction and the fabrication behaviors were discussed for bi-functional tiolor ethynyl terminated molecules.

\section{Experimental}

Figure 1 shows a schematic illustration of OMBD system and structural formula of source compounds. Cis-cis bis(ethynylstyryl)benzene (cis-cis BESB) and biphenyl-dithiol (BPDT) were used as source compounds. The growth chamber was equipped with two
Knudsen cells, which was evacuated by a turbomolecular pump. Each molecule was evaporated from the Knudsen cell in ultra-high vacuum $\left(10^{-6} \mathrm{~Pa}\right)$. The deposition rate was monitored independently for each molecular beam by oscillating quartz crystal systems and controlled by the cell temperature. Optically polished $\mathrm{KBr}$ plates were used as substrates. The substrate temperature was kept at $25^{\circ} \mathrm{C}$ during the process. The molecules on the substrates were irradiated with a $\mathrm{KrF}$ excimer laser (Lambda Physik Lextra; $\lambda=248 \mathrm{~nm}$, pulse duration $=30 \mathrm{~ns}$ ) throughout the process. The incident angle of the laser beam was $27.5^{\circ}$ to the substrate normal. The laser fluence was $2 \mathrm{~mJ} \bullet \mathrm{cm}^{-2} \bullet \mathrm{pulse}^{-1}$ at the repetition rate of $1 \mathrm{~Hz}$. FT-IR measurements were conducted in order to analyze the deposited substances (Nicolet Magna 550).

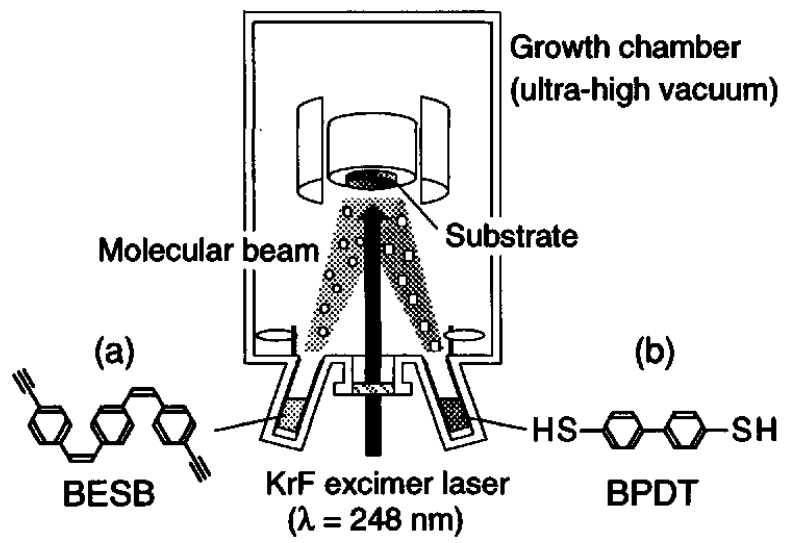

Fig. 1. The schematic illustration of OMBD system. (a) Bis(ethynylstyryl)benzene (BESB) and (b) biphenyl-4,4'dithiol (BPDT) were used as source compounds. 


\section{Results and Discussion}

Figure 2 shows FT-IR spectra of the films deposited by a single molecular beam or dual molecular beams with a $\mathrm{KrF}$ excimer laser. The deposition rate was $1.2 \mathrm{~nm} \cdot \mathrm{min}^{-1}$ for each molecule on the thickness sensor beside the substrates without laser irradiation. In the case of BPDT molecular beam alone with a $\mathrm{KrF}$ excimer laser, a film was not formed and no IR absorption peak was observed as shown in Fig. 2(a). This indicated that BPDT itself alone could not be deposited on the substrates due to its high vapor pressure even with laser irradiation. In the case of ciscis BESB molecular beam alone with a $\mathrm{KrF}$ excimer laser, the trans-trans BESB was deposited. A typical IR absorption peak attributed to $\mathrm{C}-\mathrm{H}$ bending mode of trans-vinylene was observed at $972 \mathrm{~cm}^{-1}$ as shown in Fig. 2(b). This phenomenon was explained by the finding that one-way cis-to-trans photoisomerization occurred for cis-cis BESB absorbed on the substrate surface upon $\mathrm{KrF}$ excimer laser irradiation during the deposition process.[4,5] We have reported that BESB could react intermolecularly through the terminal ethynyl groups upon the high dose UV irradiation following the photoisomerization.[6] In this case, an IR peak assigned to $\mathrm{C}-\mathrm{H}$ stretching mode of ethynyl group strongly appeared at $3276 \mathrm{~cm}^{-1}$ as shown in Fig. 2(b). Homo-polymerization did not occur under this low irradiation dose.

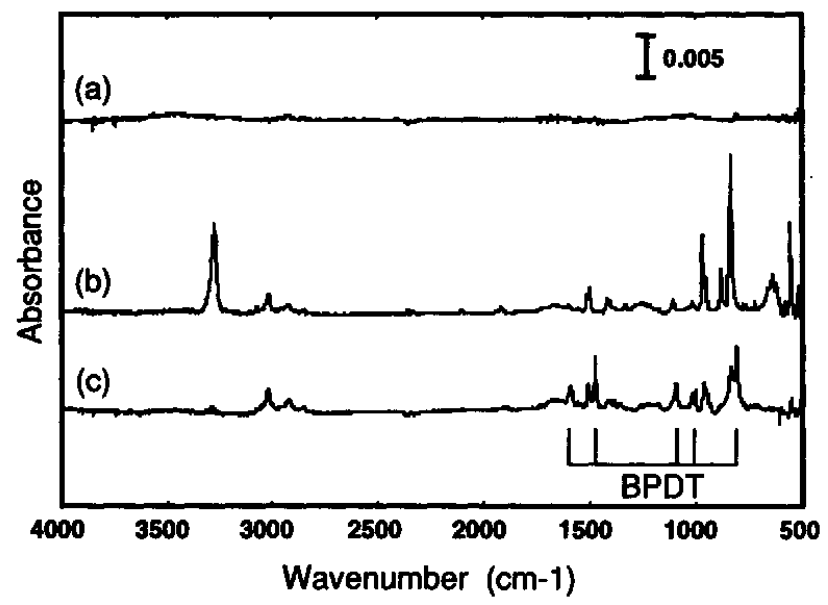

Fig. 2. FT-IR spectra of the sample deposited under various conditions; (a) the BPDT molecular beam with the $\mathrm{KrF}$ excimer laser, (b) the BESB molecular beam with the $\mathrm{KrF}$ excimer laser, (c) the dual molecular beams with the $\mathrm{KrF}$ excimer laser. The deposition rate without laser irradiation was $1.2 \mathrm{~nm} \cdot \mathrm{min}^{-1}$ for each molecule by monitoring beside the substrate.
On the other hand, in the case of dual molecular beams with a $\mathrm{KrF}$ excimer laser, IR peaks assigned to the backbone of trans-trans BESB and BPDT both appeared as shown in Fig. 2(c). This indicates that BPDT becomes to be deposited on the substrate in the case of dual molecular beams, while it can not be deposited using BPDT molecular beam alone. However, neither the IR peak of the ethynyl groups of trans-trans BESB nor the IR peak of S-H stretching mode of BPDT at $2556 \mathrm{~cm}^{-1}$ was observed in Fig 2(c). In contrast, the broad absorption appeared at $830-900 \mathrm{~cm}^{-1}$ and $900-950 \mathrm{~cm}^{-1}$, which should be assigned to a vinyl band of R-S-CH=CH-R. [7]

From these results, it is clear that only the reaction product between BESB and BPDT was deposited as the film. The intermolecular reaction is considered to occur through the functional ethynyl and thiol groups as shown in Fig. 3. The photoisomerization of BESB proceeded together with the intermolecular reaction upon the UV excitation during the deposition process, although it was not clear which reaction occurred first. Since such an intermolecular reaction as homo-polymerization could not occur between the same kind of molecules, the reaction product was assumed to be alternative co-polymer or oligomer.

The deposition process for various conditions was schematically shown in Fig. 4. BPDT was not deposited, while BESB was deposited as its trans-trans isomer in the case of each molecular beam alone with a $\mathrm{KrF}$ excimer laser (Figs. 4(a) and (b)). In case of dual molecular beams with a KrF excimer laser, the reaction product including BPDT was deposited by the intermolecular reaction as shown in Fig. 4(c). The film was optically homogeneous and showed no phase separation by an optical microscope observation.

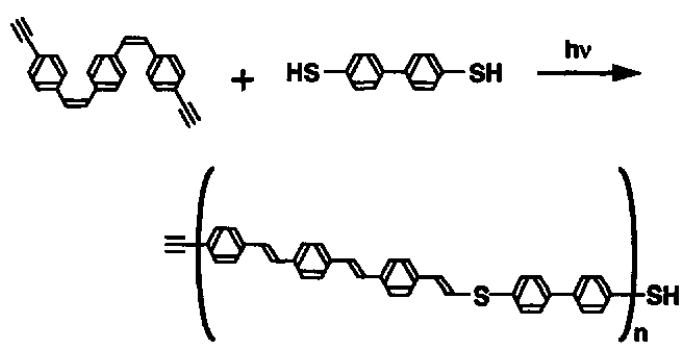

Fig. 3. Reaction scheme of BESB and BPDT upon a KrF excimer laser irradiation during the deposition process. 


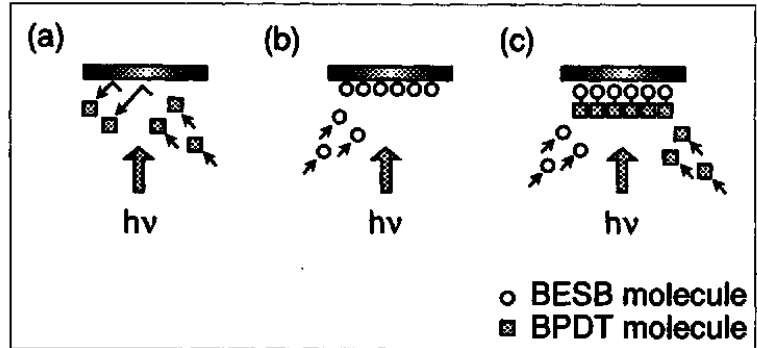

Fig. 4. The schematic illustration of the deposition process for various conditions.

We investigated the dependence of the film composition on the molecular beam intensity. The relative intensity of the molecular beams was monitored as the deposition rate on the thickness sensor without a $\mathrm{KrF}$ excimer laser. In order to measure the deposition rate of BPDT more accurately, the thickness sensor was set close to the crucible of the Knudsen cell. The deposition time was $10 \mathrm{~min}$ for all samples.

Figure 5(a) shows the relationship between BESB deposition rate and absorbance of the main IR bands of the deposited films at the constant deposition rate of BPDT. The peak intensity of BESB phenylene $(\mathrm{C}=\mathrm{C}$ stretching mode) almost linearly increased according to the deposition rate of BESB. In the re- gion of low BESB deposition rate, the peak intensity of BPDT phenylene ( $\mathrm{C}=\mathrm{C}$ stretching mode) increased with the increase of the deposition rate of BESB. In spite of the constant deposition rate of BPDT, the amount of deposited BPDT depends on the deposition rate of BESB. The IR peak of S-H stretching mode of BPDT was absent, so that only the reacted BPDT molecules were deposited. In the region of high BESB deposition rate, the peak intensity of BPDT phenylene ( $C=C$ stretching mode) was saturated. In this saturated region, the IR peak of the ethynyl groups appeared and the intensity increased with the increase of the BESB deposition rate. These results suggested that BPDT was deposited as the reaction product according to the amount of supplied BESB molecules. These support that the intermolecular reaction makes it possible for BPDT to be deposited on the substrate, as described before. On the other hand, the deposition rate of BPDT determined the upper limit of the reaction yield under the high BESB deposition rate and excess trans-trans BESB molecules, which could not react intermolecularlly with BPDT, were deposited.

Figure 5(b) shows the relationship between BPDT deposition rate and absorbance of the main IR bands of the deposited films at the constant BESB deposition rate. The peak intensity of BESB phenylene $(\mathrm{C}=\mathrm{C}$ stretching mode) was almost constant accord-

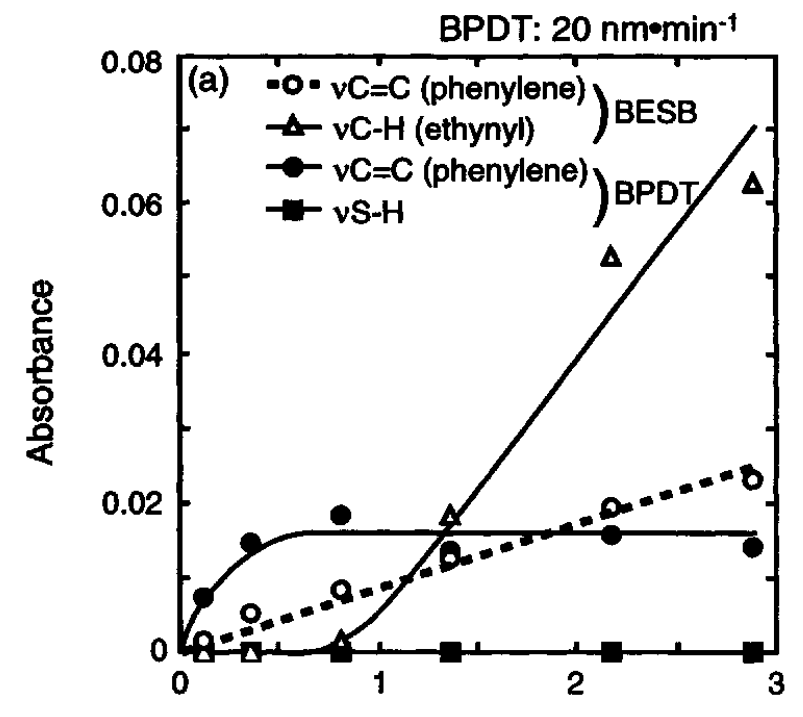

Deposition rate of BESB (nm॰min-1)

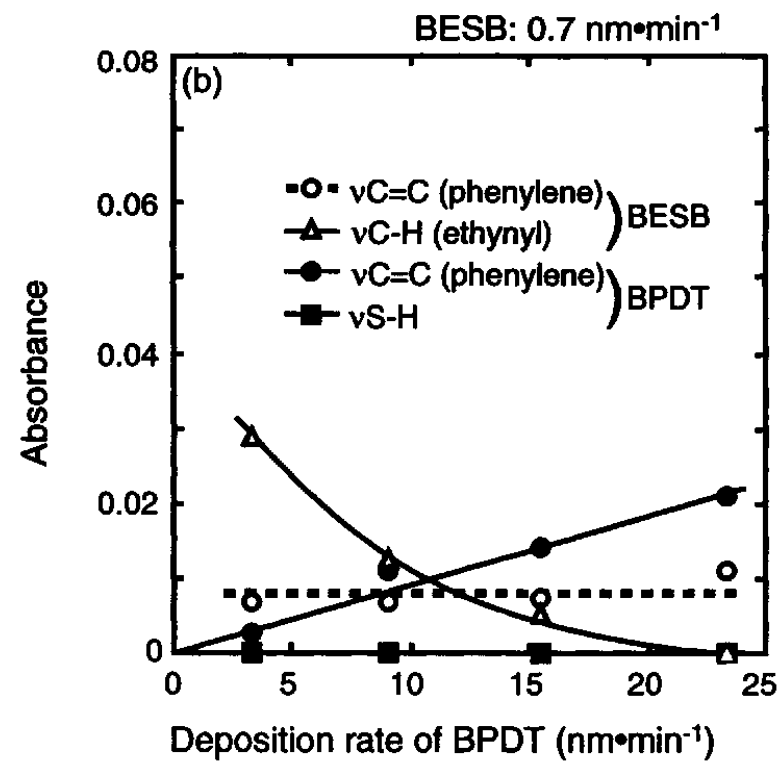

Fig. 5. The dependence of the film composition on (a) BESB deposition rate and (b) BPDT deposition rate. 
ing to the constant deposition rate of BESB. On the other hand, the peak intensity of BESB ethynyl (C$\mathrm{H}$ stretching mode) decreased with the increase of the deposition rate of BPDT. The peak intensity of BPDT phenylene ( $C=C$ stretching mode) almost linearly increased with the increase of the BPDT deposition rate. Since the peak of S-H stretching mode did not appear, only the reacted BPDT molecules were deposited as a film. Therefore, the proportion of unreacted BESB with BPDT decreased due to the proceeding of the intermolecular reaction according to the BPDT deposition rate.

From the deposition rate dependence described above, the intermolecular reaction is considered to proceed stoichiometrically. The results thus supported the deposition process simply expressed in Fig. 4.

The intensity control of each molecular beam plays an important role in the intermolecular reaction and the film composition. However, the molar ratio in the film was self-limited by the stoichiometrical reaction because the excess amount of BPDT over BESB was re-evaporated. Furthermore, the molecular structure in the films can be easily controlled by this process owing to the excellent controllability of the molecular beam intensity and the dose of the UV laser.

\section{Summary}

We investigated the thin film fabrication process based on the dual molecular beam deposition with a $\mathrm{KrF}$ excimer laser. An intermolecular reaction between BESB and BPDT proceeded through the ethynyl and tiol groups during the deposition process. The reaction made it possible for BPDT to be deposited on the substrates, which could not be deposited by the BPDT molecular beam alone. Therefore, the new organic thin films were produced by this method, which were considered to be alternative co-polymer or oligomer. The films obtained were homogeneous without the phase separation. From the dependence of the film composition on each molecular beam intensity, it was found that the intermolecular reaction proceeded stoichiometrically.

\section{Acknowledgment}

This work was conducted in the program; "Advanced Chemical Processing Technology", consigned to ACTA from NEDO, which is carried out under the Industrial Science and Technology Frontier Program enforced by the Agency of Industrial Science and Technology.

\section{References}

1. Y. Takahashi, M. Iijima, K. Inagawa and A. Itoh, J. Vac. Sci. \& Technol. A5 (1987) 2253.

2. A. Kubono, N. Kanae, S. Umemoto, T. Sakai and N. Okui, Thin Solid Films 215 (1992) 94.

3. T. Yoshimura, S. Tatsuura and W. Sotoyama, Appl. Phys. Lett. 59 (1991) 482.

4. H. Fuchigami, Y. Nakao, S. Tanimura, Y. Uehara, T. Kurata, S. Tsunoda, H. Niino and A. Yabe, $J$. Photopolym. Sci. Technol. 8 (1995) 457.

5. H. Fuchigami, Y. Nakao, S. Tanimura, Y. Uehara, T. Kurata, S. Tsunoda, H. Niino and A. Yabe, Nuclear Inst. Methods B 121 (1997) 422.

6. Y. Nakao, H. Fuchigami, A. Ueyama, Y. Uehara, T. Kurata, S. Tsunoda, H. Niino and A. Yabe, Chemistry Lett., to be submitted.

7. N. B. Colthup, L. H. Daly and S. E. Wiberley, Introduction to Infrared and Raman Spectroscopy, Academic Press (1990). 